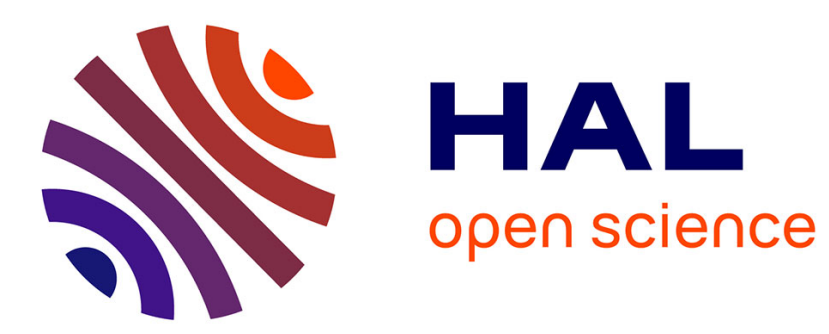

\title{
A propos des céramiques ornementales sur les édifices médiévaux du sud de la France
}

\author{
Alain Nicolaï, Lucy Vallauri
}

\section{To cite this version:}

Alain Nicolaï, Lucy Vallauri. A propos des céramiques ornementales sur les édifices médiévaux du sud de la France. Archéologie du Midi Médiéval, 1986, 4, pp.103-111. halshs-01383160

\section{HAL Id: halshs-01383160 \\ https://shs.hal.science/halshs-01383160}

Submitted on 18 Oct 2016

HAL is a multi-disciplinary open access archive for the deposit and dissemination of scientific research documents, whether they are published or not. The documents may come from teaching and research institutions in France or abroad, or from public or private research centers.
L'archive ouverte pluridisciplinaire HAL, est destinée au dépôt et à la diffusion de documents scientifiques de niveau recherche, publiés ou non, émanant des établissements d'enseignement et de recherche français ou étrangers, des laboratoires publics ou privés. 


\section{A propos des céramiques ornementales sur les édifices médiévaux du sud de la France}

In: Archéologie du Midi médiéval. Tome 4, 1986. pp. 103-111.

\section{Résumé}

En France continentale, un seul édifice ayant comporté un décor de bacini (céramique ornant les surfaces externes d'un édifice) était recensé : il s'agit de "L'Hôtel de Ville » de Saint-Antonin (Tarn-et-Garonne). La France du sud, à l'exception de la Corse, semblait ainsi s'être tenue à l'écart de ce parti décoratif qui connut, pourtant, au Moyen Age, une assez large diffusion dans le bassin méditerranéen.

Cette première enquête permet, après de nouvelles et parfois spectaculaires découvertes, comme Silvacane, Pont-Saint-Esprit, Utelle, de mieux appréhender l'aire de diffusion de ce parti ornemental. Les édifices actuellement recensés font l'objet d'une notice. Les bacini de l'église Saint- Véran d'Utelle soulèvent des problèmes complexes, plus particulièrement développés.

Citer ce document / Cite this document :

Nicolaï Alexandre, Vallauri Lucy. A propos des céramiques ornementales sur les édifices médiévaux du sud de la France. In: Archéologie du Midi médiéval. Tome 4, 1986. pp. 103-111.

http://www.persee.fr/web/revues/home/prescript/article/amime_0758-7708_1986_num_4_1_1120 


\title{
A PROPOS DES CERAMIQUES ORNEMENTALES SUR LES EDIFICES MEDIEVAUX DU SUD DE LA FRANCE
}

\author{
A. NICOLAÏ*,L. VALLAURI **
}

En France continentale, un seul édifice ayant comporté un décor de bacini (céramique ornant les surfaces externes d'un édifice) était recensé : il s'agit de «L'Hôtel de Ville » de Saint-Antonin (Tarn-et-Garonne). La France du sud, à l'exception de la Corse, semblait ainsi s'être tenue à l'écart de ce parti décoratif qui connut, pourtant, au Moyen Age, une assez large diffusion dans le bassin méditerranéen.

Cette première enquête permet, après de nouvelles et parfois spectaculaires découvertes, comme Silvacane, PontSaint-Esprit, Utelle, de mieux appréhender l'aire de diffusion de ce parti ornemental. Les édifices actuellement recensés font l'objet d'une notice. Les bacini de l'église Saint-Véran d'Utelle soulèvent des problèmes complexes, plus particulièrement développés.

In continental France, just one building on whose front can be seen a decoration of "bacini" (potteries decorating the outer surfaces of a church) was known and registered: the Townhall of Saint-Antonin (Tarn and Garonne) is only concerned. Thus, the South of France, except Corsica, seemed to have been kept apart from those designs and decors which, however, in the Middle Ages, were widely spread in the Mediterranean area.

This first inquary enables us, after recent and sometimes spectacular discoveries such as Silvacane, Pont-SaintEsprit, Utelle, to have a better approach of the area of diffusion of that ornamental system. The building which, nowadays, are known, have been, published. The "bacini " of Saint-Veran Church at Utelle, give birth to very important and new problems, but more particularly developped.

En France, le procédé consistant à utiliser des céramiques, généralement de forme ouverte (coupes, coupelles, plats...) pour décorer les surfaces externes des édifices est peu connu. Ces céramiques, animant les murs de pierre ou de brique, sont dénommées par les céramologues bacini, terme italien voulant dire bassin, employé pour la première fois dès 1758 par l'historien d'art G.B. Passeri (1). Ce type de décoration, révélé par la présence des céramiques, l'empreinte ou la trace de leur insertion sur les constructions, a été largement utilisé dans le bassin méditerranéen (2), sur les églises, plus rarement sur les édifices civils, entre les $\mathrm{XI}^{\mathrm{e}}$ et $\mathrm{XV}^{\mathrm{c}}$ siècles, pour dis- paraître presque totalement par la suite. Un des derniers bacini connu, celui de l'église Saint-Antoine de Padoue à Faenza, est particulièrement bien daté de 1702 par une inscription (3).

On les retrouve abondant en Italie (4), mais aussi en Grèce (5), quelques-uns en Espagne comme à Teruel (6), ainsi qu'en Suisse insérés sur un petit nombre d'églises du Tessin (7).

Cette ornementation céramique est à différencier des frises d'objets en terre cuite glaçurée de forme soit concave ou quadrilobée (8) que l'on recontre sur les

* Institut de Préhistoire et d'Archéologie des Alpes-Maritimes.

** Laboratoire d'Archéologie Médiévale Méditerranéenne, URA 6 CNRS.

(1) G.B. PASSERI, Istorie delle pitture in maiolica fatte in Pesaro e ne' luoghi circonvicini, Venise, 1758.

(2) J. PUIG Y CADAFALCH, La géographie et les origines du premier art roman, 1935, pp. 393-395 ; G. BERTI, L. TONGIORGI, I bacini céramici medievali delle chiese di Pisa, 1981, note 17, p. 13.

(3) H. BLAKE, The bacini of North Italy, La céramique médiévale en Méditerranée occidentale, $X^{e}-X V^{e}$ siècles, Actes du colloque international de Valbonne, 1978-1980, p. 93 et 106.

(4) Très nombreuses publications, citons parmi les plus anciennes: G. B $\Lambda$ LLARDINI, Le ceramiche del campanile di S. Apollinaire nuovo in Ravenna, Felix Ravenna 4, 1911 , p. 31 -42,150-152; id., Ceramiche architettoniche medievali, Faenza 6, 1918, p. 1-4; C. BARONI I bacini invetriati nel campanile di S. Maria Assunta a Gallarate, Rassegna Gallaratese di Storia e d'Arte 4, 1933 , p. 19 à 24. Parmi les plus récentes : F. AGUZZI, Bacini architettonici a Pavia, Atti del II convegno internationale della ceramica, Albisola, Genova 1969, p. 113-115; id., La decorazione ceramica dell'antica cattedrale di Pavia ed il problema delle prime tracce della maiolica nell'occidente cristiano, Atti.. IIL.., 1970, p. 281-293; id., I bacini di S. Teodoro in Pavia, Atti... IV... 1971, p. 317-327; G. BERTI e L. TONGIORGI, Bacini rimossi da un muro della ex-chiesa di S. Pietro di Malaventre, Pisa, Faenza 56, 27, 1970; id., Gruppo di bacini islamici di chiese romaniche pisane, Atti... IV ..., 1971, p. 295-305; id., Bacini ceramici su alcune chiese della campagna lucchese Faen $=a$ 59,41, 1973 : id. Bacini ceramici su edifici religiosi e civili delle province di Pistoia Firenze e Siena, ibid., 61, 1975, p. 123-135; id., op. cit., 1981 ; H. BLAKE, I bacini del campanile di S. Ambrogio a Varazze, Bolletino Ligustico 22, 1970, p. 130-136; id. The bacini of north Italy... op. cit., 1980 ; G. LIVERANI, I bacini del campanile di San Cassiano in Decimo a Campanio (Ravenna), Faenza 59, 1973, p. 124-127; O. MAZZUCATO, I bacini a Roma e nel Lazio, 2. Roma II. C.N.R. 1976; S. NEPOTI, I bacini in maiolica arcaia della chiesa di S. Francesco in Bologna, Faenza 54, 1973, p. 45-54; G.B. SIVIERO, I bacini della chiese ferraresi. Atti.. V..., 1972, p. 191-200; id., I bacini del campanile di Carrara S. Stefano, Padosa, Padusa 8, 1972, p. 64-78 : id., I bacini di Pozzoveggiani, Padova, Padusa 10, 1974, p. 119-129.

(5) A.H.S. MEGAW, Glazed Bowls in Byzantine Churches, Deltion of the Christian Archeological Society, IV, 1964, pp. 145-162 ; G. NICOLACOPOULOS, Céramiques encastrées des anciennes églises de Grèce, Faenza, LXIII, 1977, pp. 27-31.

(6) M. ALMAGRO, L.M. LlUBIA, La ceramica de Teruel, Teruel, 1962.

(7) J.R. RAHN, I monumenti artistici del medio evo nel cantone Ticino, 1894, p. 169 ; H. BLAKE, op. cit., pp. 93-109.

(8) D.T. RICE, Byzantine Art, Norwich, 1968, p. 107 ; C. MANGO, Architettura bizantina, Milan, 1974, p. 316; G. BERTI, L. TONGIORGI, Aspetti della decorazione con ceramiche invetriate nella architettura bizantina, Atti XII convegno internazionale della ceramica, Albisola, 1979, pp. 25-36. 


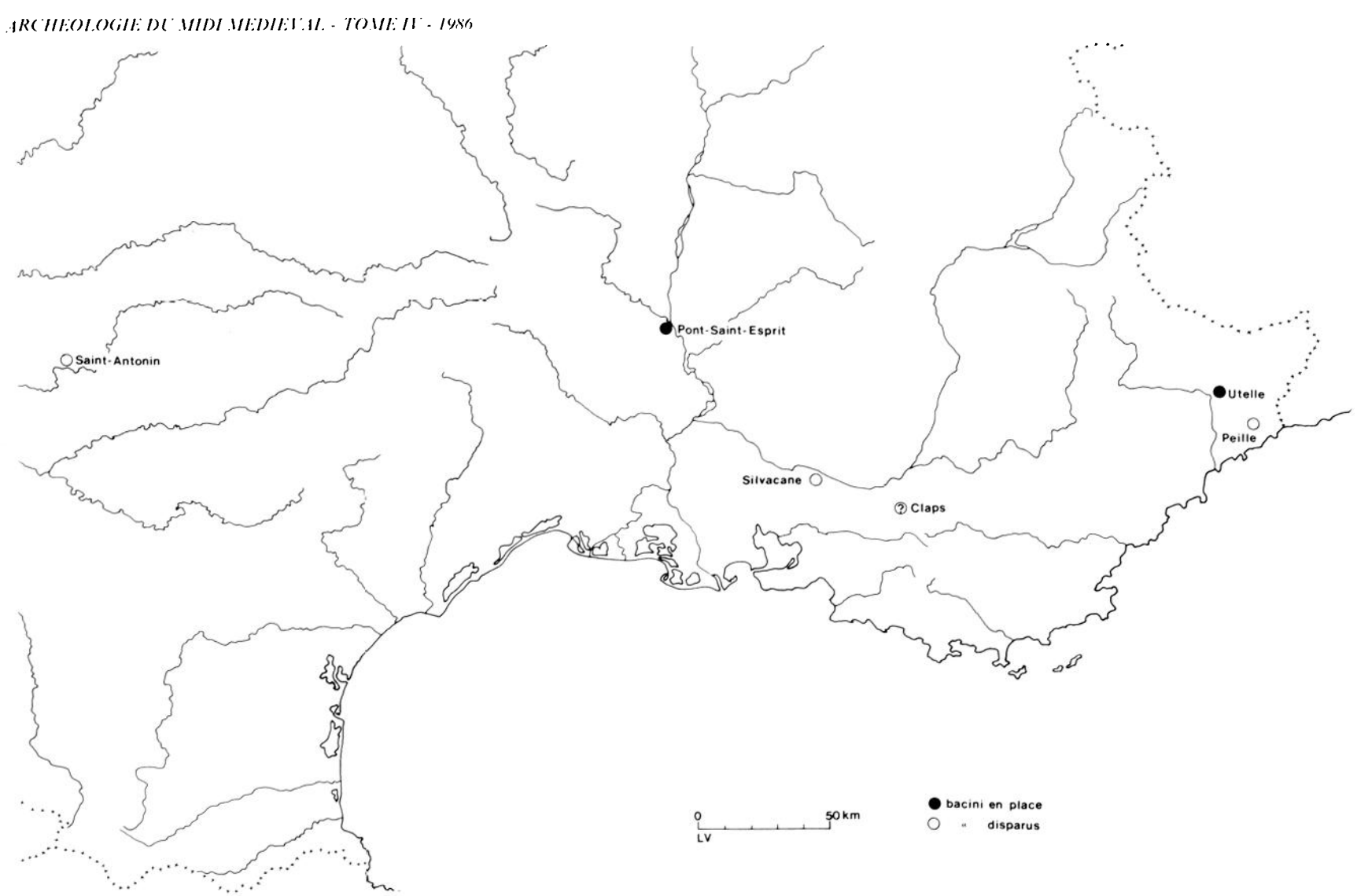

Fig. 1: Cartographie des hacini recensés.

églises byzantines de Grèce (9), de Yougoslavie (10), de Bulgaric (11), de Roumanie (12) et sur le palais de Tekfur-Sérail à Istambul (13). Ces objets en terre cuite, souvent accolés, créés spécialement pour l'édifice sur lesquel ils s'intègrent, étant le fruit d'une standardisation déjà avancée des éléments de construction.

En France, à part les onze églises corses étudiées par les spécialistes italiens, G. Berti et L. Tongiorgi (14), un seul édifice ayant comporté des bacini était connu des céramologues et des historiens de l'art: il s'agit de "l'Hôtel de Ville» de Saint-Antonin dans le Tarn-etGaronne.

On se perd en conjoncture quant à leur finalité. Prépondérance de la fonction ornementale, adjonction d'une vocation religieuse qui tenait à la fois de «la vertu prophylactique de ce qui brille et de la cupule » (15) ou, à l'origine, goût de la possession de rares objets exotiques exhibés en guise de trophée ou de don (16)? La vérité est peut-être à rechercher dans la conjonction de ces explications.

\section{ESSAI DE RECENSEMENT DES «BACINI" DANS LE MIDI DE LA FRANCE.}

En France continentale, aucune enquête systématique n'a été à ce jour entreprise (17). Un seul bâtiment ćtait connu à Saint-Antonin dans le Tarn-et-Garonne.

* Maison romane de Saint-Antonin.

Cette riche demeure bourgeoise, dont l'histoire est relativement bien cernée (18), remonte au milieu du $X I I^{c}$ siècle puisqu'on la qualifie de neuve en 1155 . Elle appartenait à la famille de Granolhet, et fut achetée en 1312 par les consuls de Saint-Antonin. La construction, composéc d'un corps de logis à deux étages, et d'une tour accolée à son extrémité méridionale, était ornée d'un fastueux décor de bacini comprenant plus d'une douzaine de céramiques. Sur la façade du logis, une série de six cavités alignées et espacées régulièrement, sćpare les deux étages, tandis que quatre autres sont situées à la retombée des grands bandeaux en demi-cercle

(9) G. MILLET. L'école grecque dans l'architecture byzantine, 1916. pp. 283-289.

(10) (j. BALS, Eglise de Serbic, Bucarest, 1911, p. 22, fig. $25:$ G. MILLET, L'ancien art serbe, les églises. 1919; C. MANGO, op. (iit. pp. 316-317 et 321-323.

(11) M. BICHEV. Bulgarian Architetture 13th-14th Centuries. Medieval Bulgarian Culture. Sofia, 1964, pp. 77-89; G. GOROV. M. LAZAROV. J. THIMBOULEVAS, Musée Archéologique de Bourgas. Sofia, 1967.

(12) Aron VODA. Buletinul comiszunii monumentelor istorice. II. Bucarest. 1909, pp. 6-9, fig. $1-4$ ct 8 : G. BALS. Beserilice lui Stefan cel Mare. Buletinul comisunii monumentelor istorice. XIX-XX. Bucarest. 1926.

(13) (j. BALS. GHIKA-BUDESTI. Ruincle bizantine din Messembria. Bulerimul comisumii monumentelor istorice. IV-V. Bucarest. $1911-1912$, p. 13.

(14) G. BERTI. L. TONGIORGI. Les céramiques décoratives sur les églises romanes de Corse. Cahiers Corsica, 53-54, 1975 , pp. $1-28$.

(15) F. BE.VOIT, La Provence e' le Comtat Venaissin. Arts et traditions populaires, réédition 1976. p. 77.

(16) T. MANNONI, La ceramica medièvale a Genova è nella Liguria. Bordighera-Gêncs, 1975. p. 172.

(17) (j. DEMIANS D'ARCHIMBAUD, M. PICON, Les céramiques médiévales en France méditerranéenne, recherches archéologiques et de laboratoire. La céramique médiérale... op., cit. pp. 359-372.

(18) M. DLRLIAT. Haut-Languedoc roman. collection Zodiaque. 1978. pp. 315-318 et pl. 146: M. DLRLIAT, L'art roman Mazenod. 1982. p. 141. 


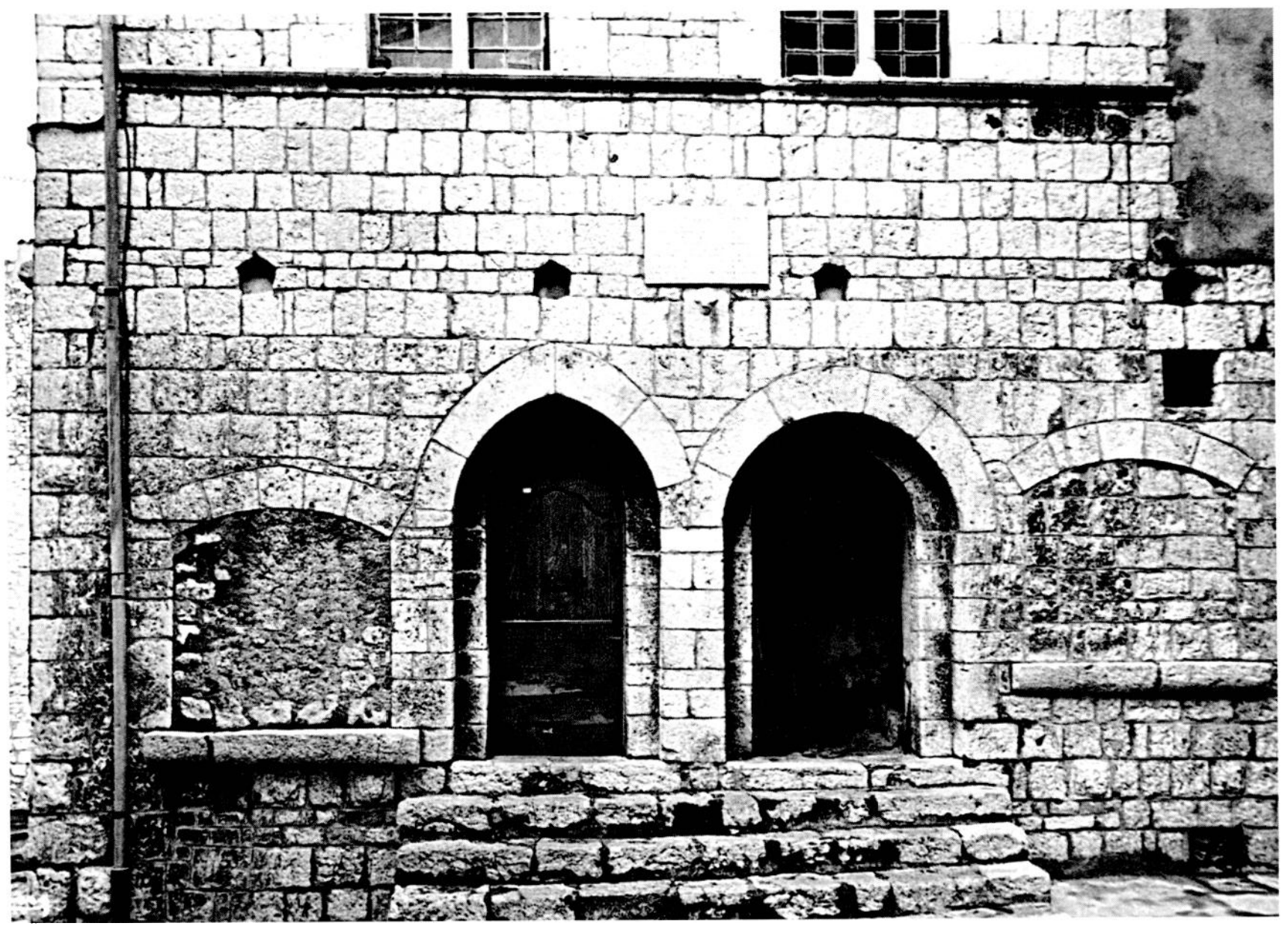

Fig. 2 : Cavités sur la façade du « Palais du Juge Mage » à Peille (Alpes-Maritimes).

qui encadrent les trois fenêtres géminées de l'étage supérieur. Une cavité est également visible, dans l'écoinçon de la baie géminée centrale. La tour, très remaniée par les restaurations de Viollet-le-Duc, ne fait pas corps avec le bâtiment, mais est datée approximativement de la même époque. Deux cavités, poursuivent l'alignement précédemment cité, de part et d'autre de la fenêtre du premier étage, unifiant ainsi le décor de la façade des deux bâtiments accolés, mais décalés en élévation.

De ce somptueux décor de faïence, il ne reste qu'un fragment conservé au musée établi sur place, et le dessin d'une écuelle, aujourd'hui disparue, décorée au lustre métallique. Celle-ci, par son décor épigraphique, floral et géométrique traité en réserve, a été attribuée aux officines de Malaga (19).

Ce parti décoratif, considéré jusqu'alors comme unique dans le Midi de la France, n'est cependant pas exceptionnel. En effet, au cours de cette recherche, plusieurs autres constructions ayant été décorées de céramiques nous ont été révélées.

\section{* Maison médiévale de Peille.}

Dans les Alpes-Maritimes, à Peille, une bâtisse civile, le «Palais du Juge Mage» (!) révèle qu’à l'origine sa fa- çade comportait un décor de bacini. Quatre cavités, de forme grossièrement pentagonales, sont alignées régulièrement séparant les deux étages de l'édifice, chacune servait de réceptable à une céramique. Elles furent découvertes, comblées de pierres en 1954 à l'occasion d'un décroûtage destiné à rendre à la façade son aspect primitif. Suivant les consignes des architectes responsables, l'ouvrier chargé à l'époque de l'opération se contenta de nettoyer les cavités tout en respectant la taille des pierres, puis il enduisit leur fond pour, selon son expression «faire propre ». Les cavités étaient taillées dans un lit de deux petites assises de pierres, chacune d'elles étant pratiquement situées au-dessus d'une des quatre ouvertures composées de deux portes d'accès, l'une en plein cintre, l'autre en arc brisé, flanquées de part et d'autre par deux larges et basses fenêtres.

En l'absence de documents, on attribue à cette construction de tradition romane, et de datation incertaine, une fonction juridique mal définie (20).

\section{* « Maison des Chevaliers » à Pont-Saint-Esprit.}

A Pont-Saint-Esprit, en Ardèche, sur un autre édifice civil, on retrouve un bacino in situ sur la façade d'une des plus anciennes maisons de la ville: «la Mai-

(19) M. DE MELY, Séance du 25 mars. Bulletin de la Société Nationale des Antiquaires de France, 1925, pp. 189-195, séance du 10 mars, pp. 153-155, 1926; G. BALLARDINI, Per un "corpus" dei bacini di ceramica di nostri antichi monumenti, Faenza, XXVI, 1938, pp. 3-16; A. LANE, Early hispano-moresque pottery: a reconsideration, Burlington Magazine, 1946, LXXXVIII, pp. 246-252, pl. l/b M. JENKINS, Medieval magribi luster-painted pottery, La céramique médiévale... op. cit., pp. 335-342, en part. fig. 28, p. 341.

(20) P. GAUBERTI, Peille, son histoire, tome I, Nice, 1966, pp. 221-222 et pl. XLIX. 
son des Chevaliers " ou " Hôtel de Piolenc », qui abrita la Cour commune de justice en 1340 (21). Une céramique à décor de lustre métallique s'inscrit dans l'écoinçon de l'ouverture géminée en plein cintre, de l'étage noble, divisée par une colonnette cannelée. Cette petite écuelle hémisphérique $(16 \mathrm{~cm}$ de diamètre) à composition radiale est ornée de feuillages stylisés traités en réserve. On connaît la difficulté de séparer les productions valenciennes des productions andalouses, en l'absence d'analyses de laboratoire (22). Aussi, aucune attribution d'origine n'est possible. On peut noter cependant que le motif végétal est proche du vocabulaire décoratif attribué à l'Espagne méridionale (23). Pont-Saint-Esprit est d'autre part situé sur le grand axe de la vallée du Rhône, où une coupe d'origine malaguenienne, attribuée à la première moitié du XIVe siècle, a été découverte au château de Beaucaire (24).

* En Provence, entre Aix-en-Provence et Vauvenargues, Fernand Benoît mentionnait la présence d'une assiette autrefois insérée dans les murs du hameau abandonné de Claps. Il soulignait, d'autre part, le rôle prophylactique que jouaient ces cupules vernissées pour la protection de la maison (25).

\section{* Abbaye de Silvacane}

Un autre édifice, mais monastique cette fois, l'abbaye cistercienne de Silvacane, construite entre 1175-1230 (26) comportait sur sa façade occidentale, au-dessus de l'occulus surmontant les trois fenêtres en plein cintre, un décor de bacini aujourd'hui disparus (27). Leur trace en négatif est encore bien conservée : on voit nettement une grosse cavité $(\varnothing \cong: 44 \mathrm{~cm})$ profonde surmontant deux petites cupules $(\varnothing \cong: 20-25 \mathrm{~cm})$ dont l'une au nord possède encore la trace de l'anneau du pied et le replat du marli. Une autre petite cavité (14 $\mathrm{cm}$ de $\varnothing$ et $5 \mathrm{~cm}$ de profondeur), sans trace de mortier est également visible, au-dessus de l'ouverture géminée de l'armarium, petite bibliothèque logée sous l'extrémi- té du bras nord du transept.

A part le bacino de Pont-Saint-Esprit, ces édifices, qu'ils soient civils ou religieux, découverts au hasard des recherches, ne conservent plus de céramiques en place. Pour cela, l'église Saint-Véran d'Utelle (AlpesMaritimes) est intéressante à plus d'un titre. Elle offre la double particularité d'être, en France continentale, et dans l'état actuel de nos connaissances, la seule église à posséder in situ un décor de bacini et de présenter le plus grand nombre de céramique en place: quatre sur les cinq primitives apparentes. Par la complexité des problèmes soulevés, l'étude des bacini d'Utelle impose un développement approfondi, objet des deux chapitres suivants.

\section{LES «BACINI » DE L'EGLISE SAINT-VERAN D'UTELLE}

Le village d'Utelle, perché à 831 mètres d'altitude domine, au nord de Nice, la vallée de la Vésubie. Une charte datée de 1150 fait mention pour la première fois de la paroisse, elle dépendait déjà de l'évêque de Nice (28). Dès 1200, l'église aurait été dédiée à Saint-Véran (29), plusieurs mentions étant encore connues pour la seconde moitié du XIVe siècle (30). L'église actuelle, postérieure dans sa conception architecturale à l'époque romane (31) (cf. plan) est, par ses amples dimensions, sa large nef à quatre travées flanquées de deux collatéraux, et ses multiples richesses décoratives sculptées et peintes, un des monuments les plus remarquables des AlpesMaritimes.

La datation et l'origine des bacini paraissent au premier abord délicates et difficiles à cerner: l'état de conservation des céramiques ne permet pas toutes les observations nécessaires à leur identification, et de plus, l'église a subi de nombreux remaniements.

Signalés dès le milieu du XIX ${ }^{\mathrm{e}}$ siècle par le baron Louis Durante (32), et attribués à l'époque romaine, ils

(21) A. GIRARD, Pont-Saint-Esprit, 1980, pp. 20-22-23. Cette façade traditionnellement datée de la fin du XIje siècle pourrait, par l'ampleur de son ouverture et la présence d'une charpente du XIV ${ }^{c}$ siècle, être plus tardive (renseignement aimablement communiqué par Y. Esquieu).

(22) G. DEMIANS D'ARCHIMBAUD, C. LEMOINE. Les importations valenciennes et andalouses en France méditerranéenne, essai de classification en laboratoire, in La céramique médiévale..., op. cit., pp. 359-372.

(23) Motif à rapprocher en particulier du bacino de l'église de San Apollinare Nuovo à Ravenna, attribué à Malaga par Manuel Gomez Moreno, cf. Jenkins (M.), op. cit., fig. 26, p. 339.

(24) G. DEMIANS D'ARCHIMBAUD, C. LEMOINE op. cit., pl. IV, fig. 9 et p. 365

(25) F. BENOIT, op. cit..

(26) A. DIMIER, L'Art cistercien, coll. Zodiaque, 1962; P. PONTUS, L'abbaye de Silvacane, Caisse Nationale des Monuments Historiques, 1982.

(27) Leur empreinte a été très exactement relevée par H. REVOIL, Architecture romane du midi de la France, Paris, 1873, tome II, pl. XIX et p. 19 - et décrite comme suit : «par dessus cette rose se trouvent encore trois petits ornements creux et arrondis, placés en forme de triangle : c'est probablement une image symbolique de la Trinité ».

Nous remercions M. Fixot d'avoir bien voulu nous signaler la présence des ces bacini. Ce dernier voyait déjà dans l'ornementation de la façade (moulurations du portail central et de l'occulus) " un sacrifice au goût du temps ", " pêché véniel » qui s'alourdit aujourd'hui par l'adjonction de trois céramiques colorées au faîte de la façade occidentale! M. FIXOT, L'excursion dans le Lubéron, Historiens, Géographes, Les Agoras Méditerranéennes, 26-29 oct. 83, n 398, 1986, p. 779.

(28) E. CAIS DE PIERLAS, Cartulaire de l'ancienne cathédrale de Nice, 1888, charte no 37, p. 48.

(29) P. CANESTRIER, Fètes populaires et traditions religieuses en pays niçois, Nice, 1978, p. 182.

(30) E. HILDESHEIMER, Utelle, d'après ses anciennes chartes, dans Nice Historique, 1952, p. 30. Dans un acte du 13 novembre 1355 , il est mentionné qu’en présence de Hugues Martini, prieur de la paroisse de Saint-Véran. des hommes du village sont réunis au presbytère pour élire pour un an le nouveau sacristain, leur choix se porte sur Antoine Janoesi. Dans son testament du 18 juin 1385, Jacques Audeberti. prieur, exprime son intention d'être enseveli dans l'église, devant l'autel. dans le tombeau de ses prédécesseurs.

(31) J. THIRION, Notes sur l'église d'Utelle. Nice Historique, 1952, pp. 35-41. Dans son analyse architecturale, l'auteur date les chapiteaux et les colonnes du XIVe siècle (?) soulignant la survivance de l'art roman, dans les Alpes du Sud, le portail, vantaux du XVIe siècle, le voûtement de l'église datant du XVII' siècle; Thevenon (L.), L'art du Moven Age dans les Alpes méridionales, Nice, 1983, p. 34.

(32) L. DURANTE, Chorographie du Comté de Nice, Turin, 1847, p. 116, "A l'angle de cette masse colossale (le clocher) s'ouvre une petite porte surmontée de trois (?) vases funéraires en terre cuite coloriée: ils paraissent dater du temps des Romains : on les trouva dans l'ancien cimetière lorsqu'on se servit de cet emplacement pour agrandir l'église ». 


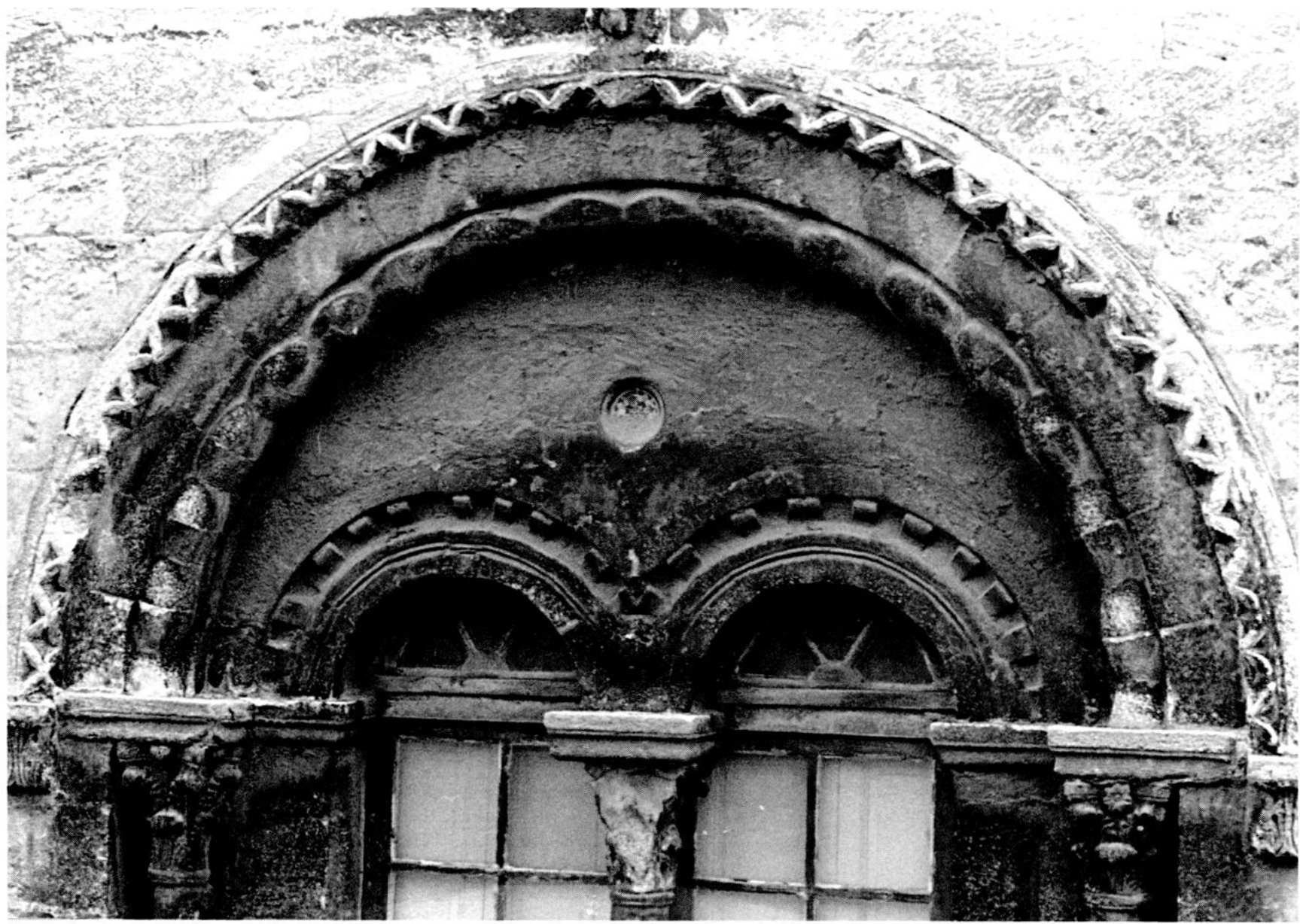

Fig. 3 a/b : Bacino ornant la façade de la Maison des Chevaliers à Pont-Saint-Esprit (Gard). Vue d'ensemble de la baie et détail de la céramique.

ont été rementionnés par J. Thirion (33) comme étant d'origine hispano-mauresque et d'époque indéterminée. Ces cinq céramiques disposées en motif crucifère (dont une a été malheureusement arrachée, il y a quelques années) s'insèrent dans le mur occidental du collatéral sud, au-dessus d'une porte en cintre brisé, et entre une fenêtre étroite et haute en plein cintre.

Les quatre exemplaires conservés présentent des caractères communs. Ils sont tous de dimensions moyennes $(\varnothing$ variant de 19,5 à $23 \mathrm{~cm}$ ) et ont une faible concavité $(4$ à $5 \mathrm{~cm}$ ). Ils se différencient cependant par leur technique décorative et leur variante de profil ; les extérieurs décorés (?) n'ont pu être malheureusement observés. Trois bacini de forme légèrement carénée, à petite lèvre recourbée vers l'extérieur ( $\left.n^{\circ} 1\right)$ ou aplatie ( $n^{\circ} 2$ et 4) possèdent un décor peint, sur un fond d'émail stannifère jaunâtre, au brun de manganèse rehaussé de larges aplats verts d'oxyde de cuivre. Un seul se distingue par son absence de décor $\left(n^{\circ} 3\right)$, sa couverte monochrome verte et son large marli. Occupant la place centrale du motif crucifere, la coupe $n^{\circ} 1$ (dont une partie a été détruite), offre un décor rayonnant de quatre fuseaux entre lesquels s'inscrivent des motifs en écoinçon sur fond d'émail stannifere variant du blanc au jaune. Des bandeaux bruns scandent régulièrement la lèvre. Le fond visible par la cassure, est un piédouche ; et la pâte utilisée de couleur rouge vif. De part et d'autre du précédent

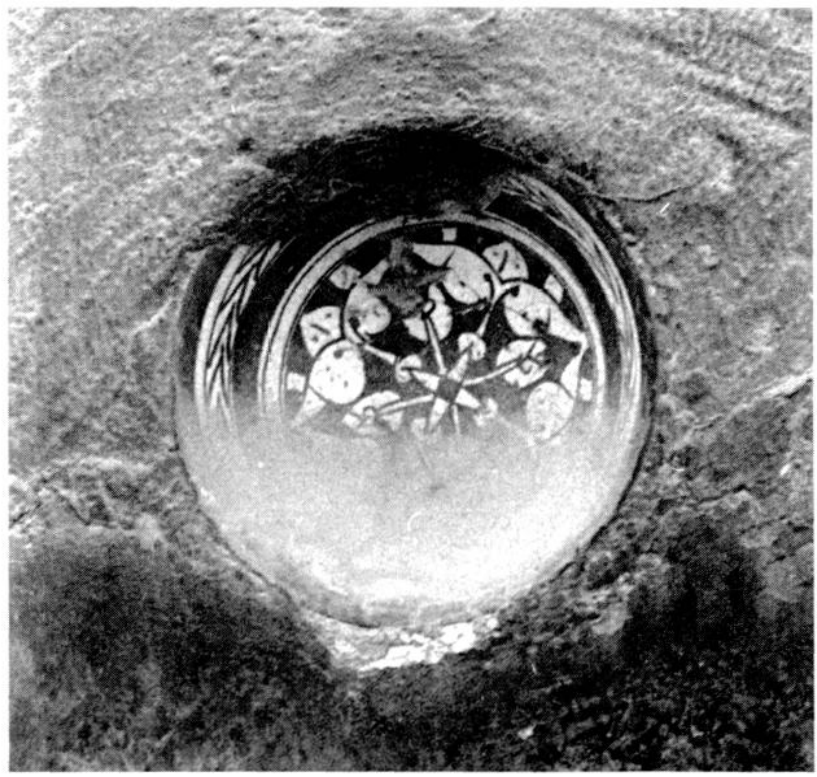




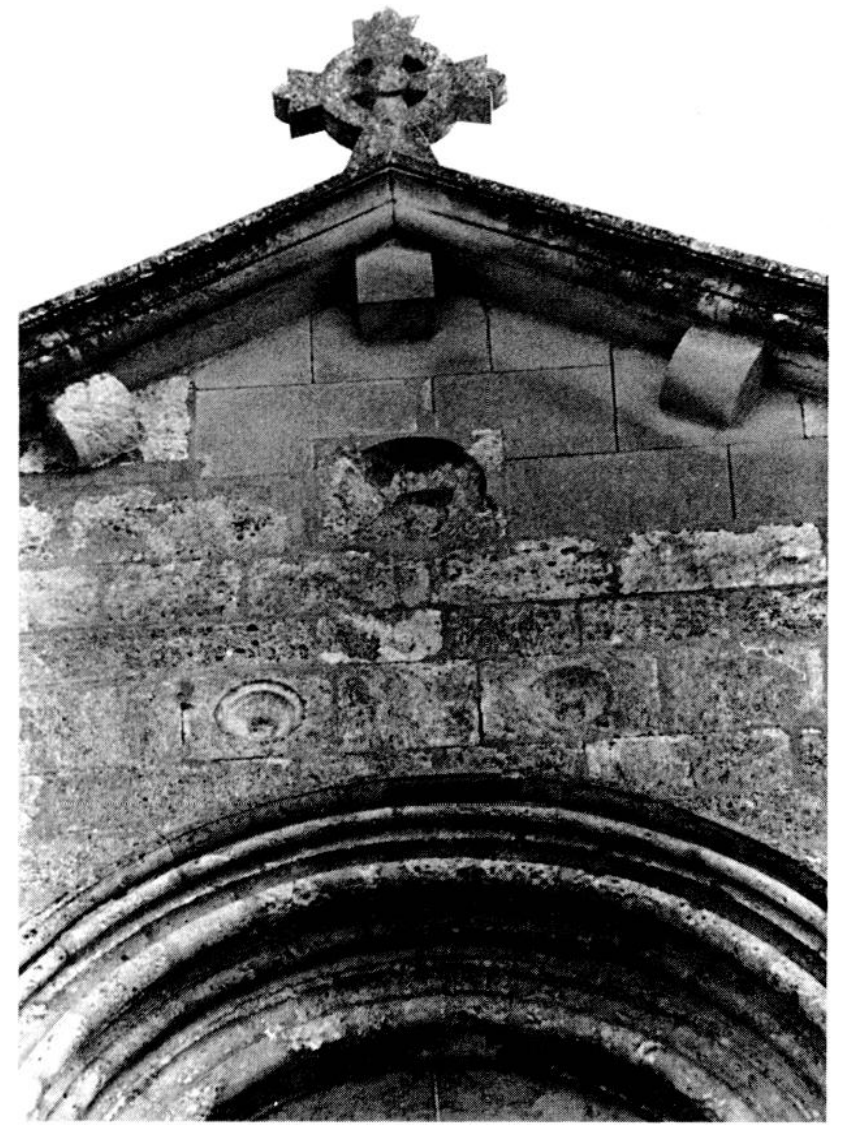

Fig. 4 : Cavités sur la façade de l'abbaye de Silvacane (Bouchesdu-Rhône).

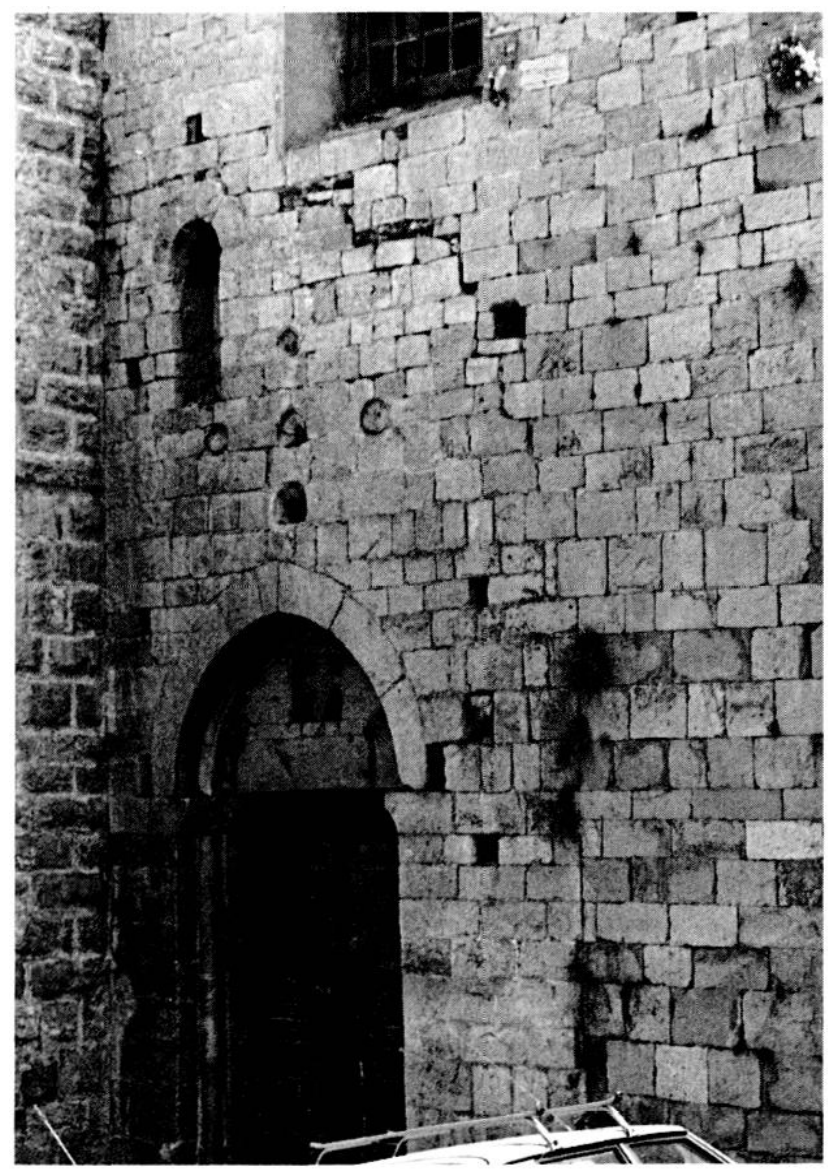

Fig. 6 : Bacini sur la façade occidentale de Saint-Véran d'Utelle.

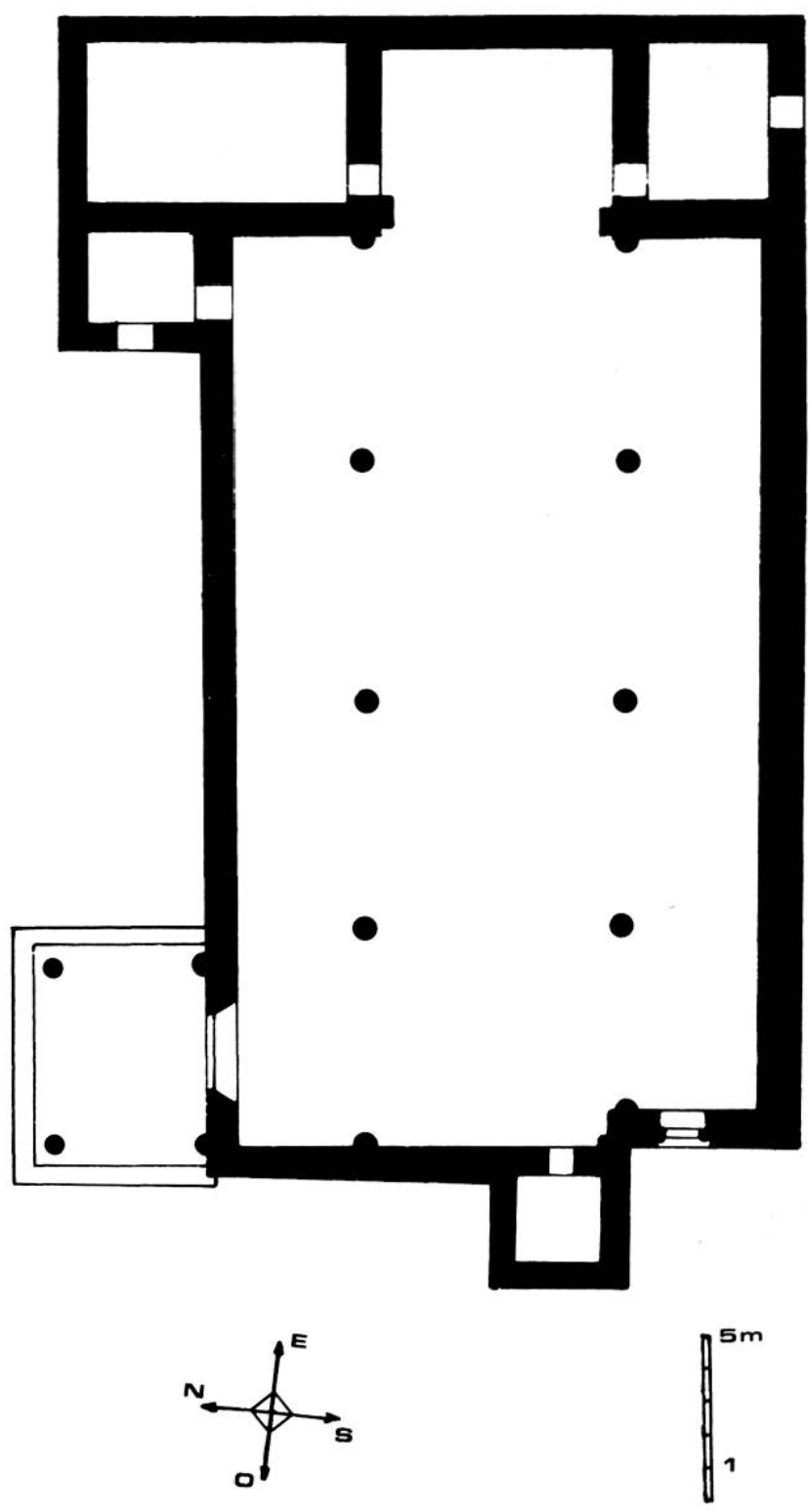

Fig. 5: Plan sommaire de l'église d'Utelle d'après le relevé effectué en 1949, par les Bâtiments de France, éch. $1 / 100^{e}$.

bacino, les coupes 2 et 4 présentent un décor axial. Une large bande verte cernée de manganèse sépare les céramiques en deux zones symétriques remplies, pour le $\mathrm{n}^{\circ} 2$ de motifs ovales et de motifs en bandes pseudoépigraphiques pour le $n^{\circ} 4$. De couleur de pâte identique, beige-rosé, ces coupes en meilleur état de conservation ne laissent pas entrevoir la forme des pieds (sans doute annulaire). Leurs lèvres semblent avoir également été décorées de bandeaux au brun de manganèse. Bien conservé l'exemplaire monochrome, à la pâte rouge recouverte de glaçure verte et taches plus sombres, occupe la partie supérieure du schéma décoratif. Peut-être fautil imaginer une céramique identique dans la partie inférieure du motif crucifêre où seul un fragment à pâte rouge, marqué par des traces d'oxyde de cuivre subsiste dans la cavité.

La structure légèrement carénée des profils des bacini $n^{\circ} 1,2,4$ et les décors pseudo-épigraphiques ( $n^{\circ}$ 4 ) et en amande ( $\left.n^{\circ} 2\right)$ renvoient aux répertoires stylistiques des poteries islamiques polychromes découvertes sur les sites archéologiques du Maghreb (CarthageByrsa), de la Sicile (Syracuse) et de la Corse (Mariana) 
(34). Des références plus précises se retrouvent dans le groupe maghrébin-sicilien (mal séparable), récemment mis en lumière, dans l'ouvrage de synthèse sur les baci$n i$ des églises de Pise (35). La grande quantité de matériel parvenu dans cette ville laisse supposer un ou plusieurs ateliers de fabrication importante et de grande diffusion.

Bien qu'aucun exemplaire du corpus ne soit parfaitement identique, ni dans la forme, ni dans les décors, il paraît possible de rapprocher les exemplaires 1, 2, 4 des formes fig. $63,65,70$, pp. $177,179,180$, les décors à organisation axiale, et décor vert en larges bandes et décor d'amande des planches LXXV-LXXVII, et ceux à organisation radiale et décor végétal stylisé des planches LXXVI et LXXIX. L'exemplaire monochrome à marli, à la glaçure verte très dense, est aussi à rapprocher des productions islamiques, où fréquemment le centre de la coupe est imprimé de cercles concentriques (cf. fig. 142, p. 221).

La datation de ces types de céramiques islamiques émaillées et vernissées donnée par les bacini pisans en fonction des édifices sur lesquels ils s'insèrent, reste haute - première moitié du XIe siècle - premier quart du XII' siècle -, des écuelles monochromes vertes identiques sont datées plus tardivement du XIII ${ }^{e}$ siècle par T. Mannoni (36). De même, cet auteur propose une datation de la seconde moitié du XII ${ }^{e}$ siècle et du début du XIII ${ }^{e}$ siècle pour une écuelle retrouvée en fouille à Gênes (San Silvestro) (37). Elle ressemble, tant par le décor que par la forme, à l'exemplaire no 4 d'Utelle à décor épigraphique.

L'identification des céramiques d'Utelle, et leur rapprochement aux productions islamiques des $\mathrm{XI}^{e_{-}}$ XIII' ${ }^{\mathrm{e}}$ siècles, à l'évolution chronologique et stylistique mal connue, posent cependant le problème de leur insertion dans l'église Saint-Véran, et de la datation du mur occidental du collatéral sud.

\section{ESSAI D'ANALYSE ARCHITECTURALE DU MUR OCCIDENTAL DE L'EGLISE D'UTELLE}

Aucune étude architecturale complète de l'église Saint-Véran n'a été entreprise à ce jour. Les recherches antérieures ont surtout porté sur l'analyse des colonnes, des chapiteaux, et des parties tardives élevées à partir de la fin du $\mathrm{XV}^{\boldsymbol{c}}$ siècle. L'implantation et la genèse de ou des édifices antérieurs n'ont jamais été abordés, sans doute à cause de la difficulté d'interprétation des rares vestiges encore en place. Seules des fouilles, ou des travaux de restauration, permettraient de recueillir des renseignements sur les fondations et la disposition de la construction primitive. Il n'est donc pas dans notre propos de reprendre l'ensemble de l'étude architecturale, mais, plus modestement, d'essayer de dégager, en fonction des éléments visibles actuellement, des données qui nous permettent de mieux cerner la datation du mur occidental du collatéral sud où sont insérés les bacini.

On remarque sur cette partie de l'église un décrochement: J. Thirion se demandait s'il s'agissait de la trace d'une réparation maladroite ou la marque de l'insertion de la porte dans un mur plus ancien. Une observation attentive à la lumière rasante permet de se rendre compte que cette fissure se prolonge à partir du haut de la baie en plein cintre jusqu'aux assises. On pourrait penser que cc coup de sabre est la conséquence d'un mouvement du bâtiment, provoqué peut-être par un tremblement de terre, si fréquent dans la région (38). Cependant, le long du décrochement, pratiquement à la hauteur des corbeaux de la porte, on voit un moellon taillé en crochet qui montre assurément que cette hypothèse ne peut être retenue. Ce bloc atteste, ainsi que la non-correspondance des lits des joints, de la noncontemporanéité des murs existants de part et d'autre du décrochement, l'un ayant nécessairement été édifié à partir de l'autre. A l'évidence, le mur extérieur a été monté avec les blocs de réemploi, sur le mur intérieur sur lequel il s'appuie. Ce mur, qui supporte la porte, la baie, et les bacini, forme un ensemble homogène témoin d'une église antérieure.

Un des éléments les plus significatifs pour sa datation doit être la porte avec son archivolte en cintre brisé. Son tympan, percé d'une ouverture ornée d'une croix, est encadré par un cordon taillé à trois facettes, il repose sur deux corbeaux moulurés d'un cavet. J. Thirion la date approximativement du XIVe siècle (39), en fait, des portes de forme identique se retrouvent sur des églises du XIII ${ }^{e}$ siècle, comme la cathédrale de Grasse ou Notre-Dame-du-Plan et Saint-André du Roc à Castellane, sur une église de la fin du XII ${ }^{c}$ ou de la première moitié du XIII' siècle : Saint-Victor de Castellane (40).

Sur la façade occidentale de Saint-Véran, au nord du clocher tardif (XVI ${ }^{-}$-XVII ${ }^{\text {e }}$ siècle), on remarque les traces d'une porte similaire. De celle-ci, obturée aujourd'hui, il ne reste qu'un piédroit et la moitié du cintre. Plus haut, on distingue la trace d'une ouverture. A droite, un net coup de sabre indique clairement que l'actuel collatéral nord a été agrandi à partir d'un édifice de proportion plus restreinte. En fonction de ces éléments, il aurait été tentant de supposer que la façade, à une époque indéterminée, comportait deux portes latérales surmontées de baie, ou que ces deux portes en encadraient une autre comme par exemple à la cathédrale de Grasse. Cependant, les deux murs ne sont pas dans le prolongement l'un de l'autre, comme on peut s'en rendre compte de l'intérieur et sur le plan très précis relevé par les Bâtiments de France en août 1949. On constate aussi que leur appareil n'est pas identique, celui de la porte murée étant moins soigné, sans doute postérieur.

La complexité des remaniements de la façade occidentale déjà souligné par $J$. Thirion et éclairée d'un jour nouveau par ces récentes observations architecturales, n'est qu'un argument supplémentaire en faveur d'une

(34) Voir à ce sujet, la synthèse de G. DEMIANS D’ARCHIMBAUD, La Céramique médiévale, les fouilles de Mariana (Corse), Cahiers Corsica, 1972, pp. 10-11.

(35) G. BERTI, L. TONGIORGI, 1981, op. cit,, cf. l'ensemble du chapitre consacré aux céramiques vernissées et émaillées islamiques de production magrébine et sicilienne, p. 162 et suivantes.

(36) T. MANNONI, op. cit., 1975, pp. 54-55, fig. 45, type 40 ; I. et D. CABONA. A. GARDINI, T. MANNONI, M. MILANESE, Contributi dell'archeologia medievale ligure alle conoscenze dei prodotti ceramici nel mediterraneo occidentale, in La céramiqué Médiévale..., op. cit., p. 118 , fig. 15.

(37) T. MANNONI, op. cit., 1975, type 82, pp. 104, 106, fig. 90, 183

(38) Sous la direction de J. VOGT, Les tremblements de terre en France, Mémoires du B.R.G.M., n० 96, 1979, article de (adiot (B.). pp. 107-122.

(39) J. THIRION, op. cit., 1952, p. 36.

(40) J. THIRION, Alpes romanes, 1980, pp. 49, 197-205, fig. 53, 335-340; Thirion (J.), Les églises romanes de Castellane, in Annales de Haute-Provence, $\mathrm{n}^{\circ} 294,1982$, pp. 149-171. 

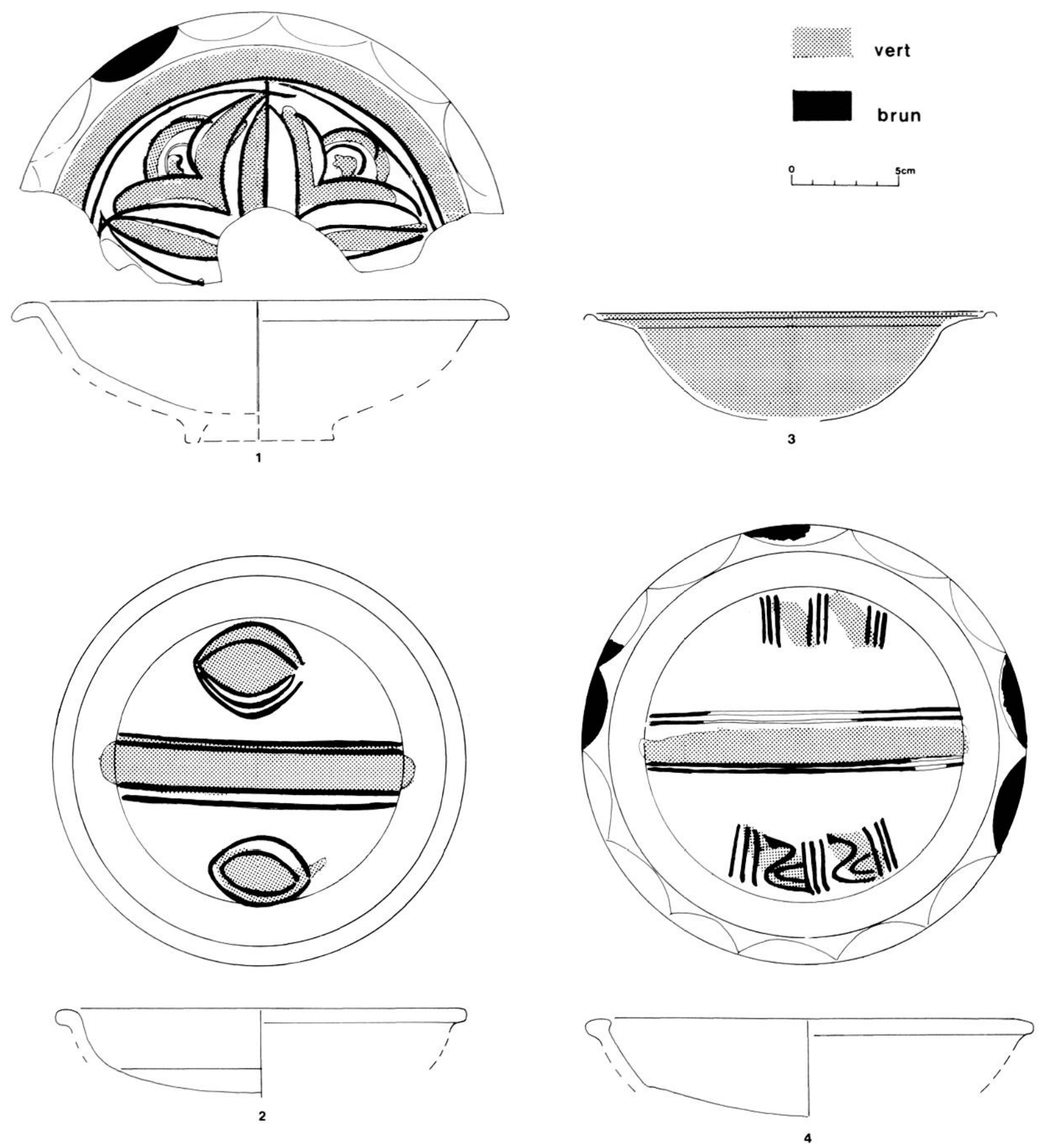

Fig. 7 : Bacini de la façade occidentale de Saint-Véran d'Utelle, éch. 1/3.

datation relativement ancienne des bacini d'Utelle. Le mur supportant les bacini reste peut-être avec ces céramiques les seuls témoins d'un (premier) édifice roman du XIII' siècle.

En conclusion, l'ensemble des bacini cités dans ces notes permet, d'une part, de mieux cerner les courants d'échanges avec le monde méditerranéen au Moyen Age et, d'autre part, de mieux définir l'aire de diffusion de ce procédé ornemental. Si la présence de bacini sur un mur de l'église d'Utelle et la façade du Palais de Peille s'explique facilement par la proximité de l'Italie, on reste plus surpris par des découvertes de la Provence et de la vallée du Rhône (Silvacanc, Pont-Saint-Esprit). Le cas jusqu'alors isolé de Saint-Antonin dans le Tarn-etGaronne ne fait désormais plus exception. La France du Sud semble, comme tous les pays méditerranéens, avoir connu le même engouement pour ce mode décoratif. Dès lors, l'aire de diffusion des bacini paraît avoir été nettement plus occidentale et septentrionale qu'on ne le supposait. Ce premier recensement non exhaustif nous autorise à penser que d'autres édifices civils ou religieux ornés de céramiques, restent à découvrir. Il serait d'autre part souhaitable que ce patrimoine, fragile mais remarquable, lorsqu'il a eu la chance d'être conservé in situ (comme à Utelle ou Pont-Saint-Esprit), fasse l'objet de mesures de protection, à l'exemple des interventions italiennes. 


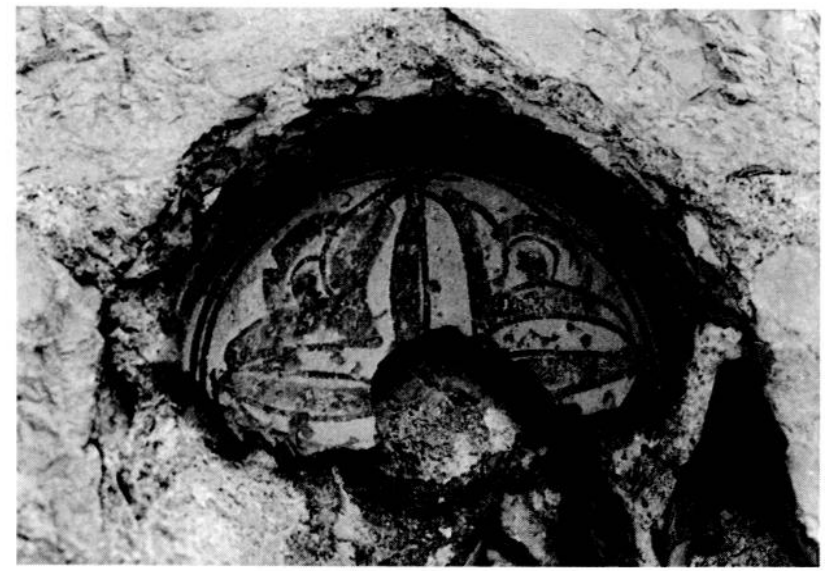

a

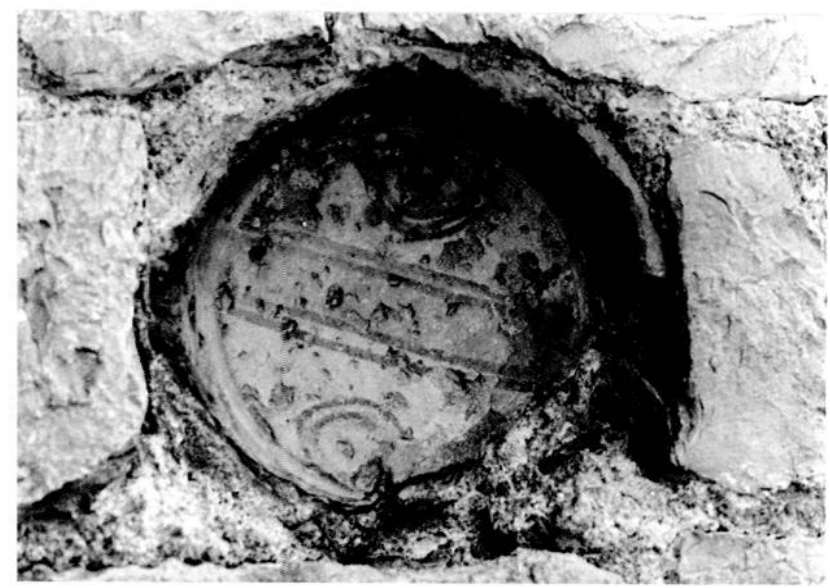

b
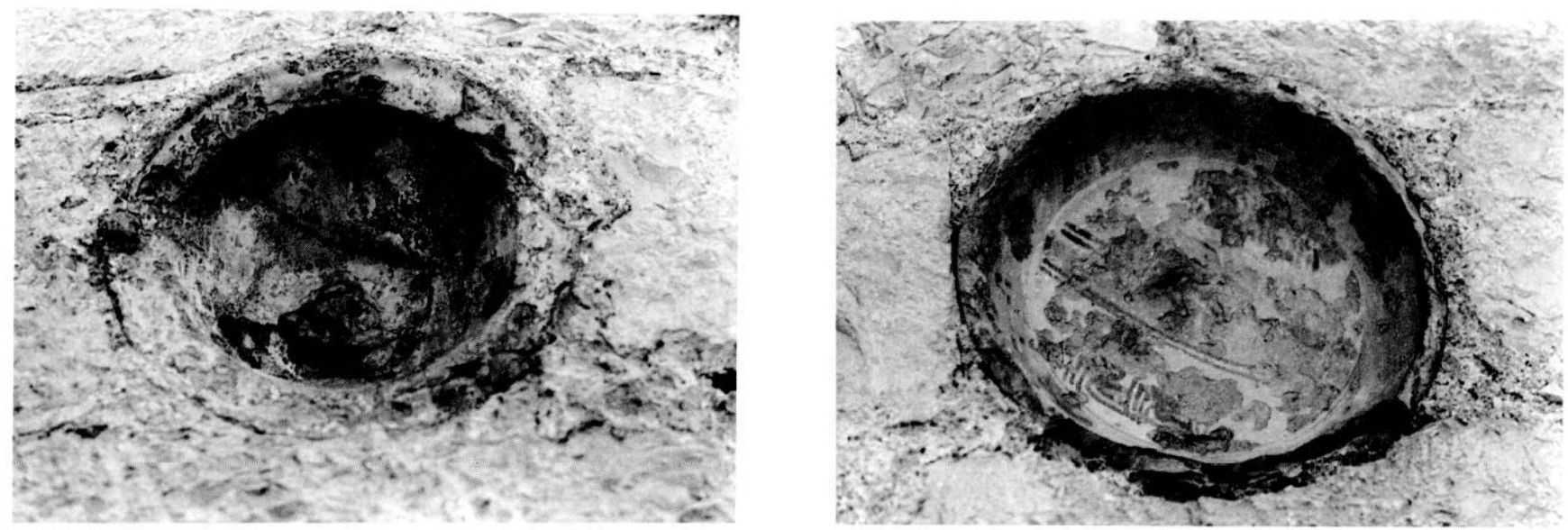

Fig. $8:$ idem fig. $7: a=1, b=2, c=3, d=4$.
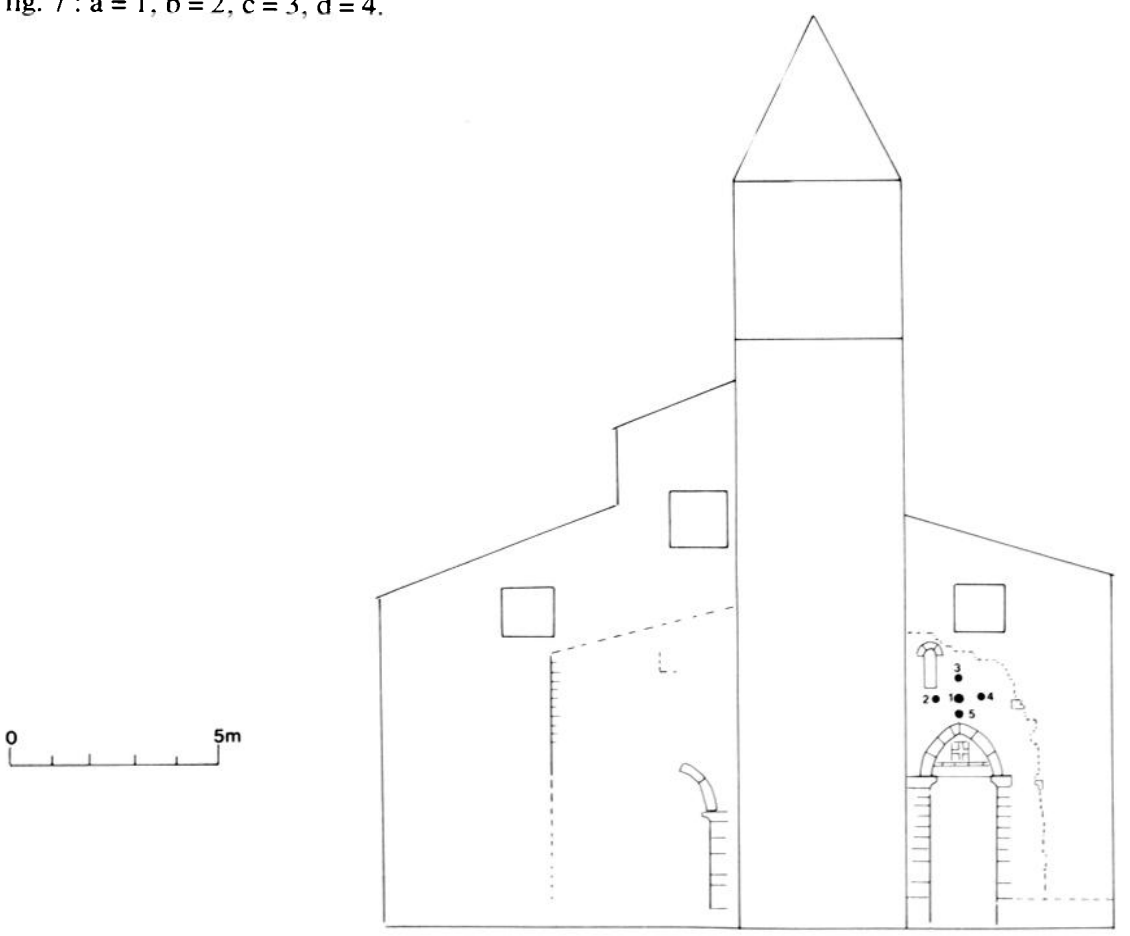

Fig. 9 : Croquis de la façade occidentale de l'église d'Utelle. 